\title{
Altered forefoot function following a military training activity
}

H. Rice ${ }^{1}$, J. Fallowfield², A. Allsopp ${ }^{2}$, S. Dixon ${ }^{1}$

${ }^{1}$ School of Sport and Health Sciences, University of Exeter, Exeter, UK ${ }^{2}$ Institute of Naval Medicine, Alverstoke, Gosport, PO12, UK

Corresponding Author Contact Details:

Hannah Rice:

H.Rice@exeter.ac.uk

01392724722

Sport and Health Science

Richards Building

St Luke's Campus

Heavitree Road

Exeter

EX1 2LU, UK

\section{Conflict of Interest Statement}

We are not aware of any conflict of interest related to the manuscript and its publication.

This work was part-funded by the Institute of Naval Medicine.

(C) Crown Copyright (MOD) 2019 


\section{Abstract}

Background: Injury rates are high in populations that regularly undertake weightbearing physical activity, particularly military populations. Military training activities, that often include load carriage, have been associated with lower limb injury occurrence, specifically stress fractures.

Research question: Recent work identified plantar loading variables as risk factors for lower limb stress fractures in Royal Marines recruits that were assessed during barefoot running. This study aimed to quantify how those plantar loading variables changed in Royal Marines recruits following a prolonged military load carriage activity, to further understand potential mechanisms for lower limb stress fractures.

Methods: Bilateral, synchronised plantar pressure and lower limb kinematic data were recorded during barefoot running at $3.6 \mathrm{~m} \mathrm{~s}^{-1}( \pm 5 \%)$ pre- and post- a 12.8-km training activity ( 150 min). The training activity was completed with an average speed typical of walking $\left(1.4{\mathrm{~m} . \mathrm{s}^{-1}}^{-1}\right.$, and $35.5 \mathrm{~kg}$ of additional load was carried throughout. Data were collected from 32 male Royal Marines recruits who completed the training activity in week-21 of the 32-week training programme. Plantar pressure variables and ankle dorsiflexion were compared between pre- and post-activity.

Results: Post-activity there was reduced loading under the forefoot and increased loading under the rearfoot and midfoot. There was no change in dorsiflexion touchdown angle, but an increase in peak dorsiflexion and range of motion postactivity.

Significance: The increased rearfoot loading, reduced forefoot loading and increased ankle dorsiflexion following a prolonged military load carriage activity suggest a reduced transfer of loading from the rearfoot to the forefoot during stance, which may 
Accepted in Gait and Posture, September 2019, https://doi.org/10.1016/i.gaitpost.2019.09.009

have implications for the development of stress fractures, particularly of the metatarsals.

Keywords: stress fracture; plantar pressure; load carriage; military training 


\section{Introduction}

Injury rates are high in active populations who regularly undertake vigorous weightbearing physical activity, and are known to be higher amongst military recruits than distance runners [1]. The Royal Marines recruit population has a high injury rate during initial training (16\% [2]), with stress fractures of the metatarsals and tibia among the most common injuries [3]. The 32-week recruit training programme includes frequent, prolonged military training activities, often whilst carrying load. Military training activities have previously been associated with lower limb injury occurrence in military recruits [4-6] and specifically with the high stress fracture occurrence in the Royal Marines recruit population [7]. Within this population, a review of the training programme resulted in a revised syllabus, which in turn was associated with a reduction in stress fracture incidence [7]. Nonetheless, the training programme remains necessarily demanding and stress fractures continue to be a problematic injury, due in part to their lengthy recovery time [3]. Understanding the influence of military training activities on lower limb loading is therefore warranted.

Stress fractures are the result of excessive, repetitive stress on a bone [8], which may explain their prevalence in populations who frequently participate in weight-bearing, endurance activities. The mechanisms by which military training activities may exacerbate stress fracture risk are presently unclear. Load carriage during walking results in increased second metatarsal compression [9], which can increase bone damage accumulation [10]. Metatarsal loading was increased following running [11][15], whereas reduced metatarsal loading was observed after four consecutive days of long-distance walking (160 - $200 \mathrm{~km}$ total) [16]. Reduced loading under the lesser 
toes $[12,13,15,16]$ and hallux $[11,13,16]$ was also observed after walking and running. This shift of loading from the toes to the metatarsals during prolonged activity may be associated with the relatively high stress fracture risk in populations who regularly undertake such activities.

Recent prospective studies of Royal Marines recruits have identified characteristics exhibited during barefoot running that predispose a recruit to a second or third metatarsal stress fracture [17] or a tibial stress fracture [18] during the 32-week recruit training programme. A lower dynamic arch index (i.e. higher arch, assessed as percentage of plantar-ground contact in the midfoot area) and later occurrence of peak pressures during stance under the metatarsal heads were identified as risk factors for second and third metatarsal stress fractures respectively. Greater peak pressure under the heel was identified as a risk factor for tibial stress fracture [18]. In order to further understand why these characteristics may predispose to risk of site-specific stress fractures it is important to understand the influence of the physically demanding training activities on these characteristics. Changes to plantar loading following a military load carriage training activity are unknown. Given the association between load carriage activities and stress fractures within this population, altered plantar loading following such an activity may explain mechanisms for the development of stress fractures. Quantifying changes to those variables that have previously been identified as risk factors for stress fracture is therefore important. Assuming that a recruit is in sub-optimal physical condition following a demanding training activity, a tendency to demonstrate characteristics associated with stress fracture may be expected following the activity. 
The aim of this study was to investigate changes to plantar loading assessed during barefoot running following a prolonged load carriage training activity in Royal Marines recruits. It was hypothesised that there would be reduced loading under the toes and hallux, accompanied by increased metatarsal loading post- compared with pre-training activity. It was also hypothesised that peak pressures under the metatarsals would occur later during the stance phase and that peak pressure under the heel would increase post- compared with pre-training activity.

\section{Methods}

32 male Royal Marines recruits volunteered to participate in the study, which was undertaken in week-21 of the 32-week recruit training programme. All volunteer recruits were injury-free and had completed the required training up to the point of data collection without being removed from mainstream training for any reason. Within these criteria, recruits were randomly selected from three different training troops who had followed the same training programme. Volunteer recruits had a mean (SD) age: 22.19 (3.84) years, body mass: $82.4(8.4) \mathrm{kg}$, height $1.78(0.06) \mathrm{m}$, and body mass index (BMI): 25.9 (2.0) kg.m-2. Recruits had completed 20 weeks of training prior to data collection and as such were relatively homogeneous in terms of training status. The study was approved by the Ministry of Defence Research Ethics Committee (MODREC Reference 367/Gen/12), and all recruits provided informed, written consent.

As part of the Royal Marines recruit training programme, all recruits are required to cover $12.8 \mathrm{~km}$ on foot, whilst carrying a load of $35.5 \mathrm{~kg}$, in week-21 of the training programme. This study collected running data pre- and immediately post- this military 
training activity during the recruit training programme. The training activity took place on uneven terrain at varying paces and modes (walking and running), with an approximate average duration of 150 minutes. This equates to an average speed of $5.1 \mathrm{~km} . \mathrm{h}^{-1}\left(1.4 \mathrm{~m} \cdot \mathrm{s}^{-1}\right)$. The training team determined the pace of the activity, with all recruits required to complete the activity together. Recruits wore military issue clothing and boots throughout the activity. There was a pause in the activity approximately every hour for water breaks, and recruits were encouraged to eat regularly throughout the activity. The load consisted of a bergen (large military rucksack) and webbing (worn like a belt to carry additional military equipment) with a combined mass of 31.3 $\mathrm{kg}$, and a weapon $(4.2 \mathrm{~kg})$.

Bilateral, synchronised plantar pressure and lower limb kinematic measurements were recorded during barefoot running at $3.6 \mathrm{~m} . \mathrm{s}^{-1}( \pm 5 \%)$. This barefoot running protocol has previously been used in studies identifying risk factors for lower limb injuries in Royal Marines recruits $[17,18]$. By administering the same protocol it was possible to determine whether the variables associated with greater risk of stress fracture in this earlier, prospective study were altered following the demanding military training activity. Evidence that gait characteristics are reportedly more pronounced when barefoot than shod influenced the selection of barefoot data collection for this task [19].

Sagittal plane ankle kinematic data were collected to assist in understanding any observed changes in plantar loading. Kinematic data were collected with two Coda Mpx30 units at $200 \mathrm{~Hz}$ (CodaMotion, Charnwood Dynamics, UK) using eleven active markers per leg, and secured using Micropore ${ }^{\mathrm{TM}}$ tape (3M, USA). Recruits wore shorts 
and t-shirt during the pre- and post-activity running trials. Marker positions were identified with pen to allow reliable replacement following the training activity. Plantar pressure was recorded using a 2 m pressure plate (RSscan International, Belgium, 4 x $0.5 \mathrm{~m} \times 0.4 \mathrm{~m}, 4096$ sensors, $125 \mathrm{~Hz}$ ) positioned and marked in the middle of a 10 m EVA runway.

Recruits were asked to complete running trials with a relaxed running style, ensuring that two consecutive steps contacted the plate. They used a self-selected foot strike and order of foot placement. Recruits completed familiarisation trials until successful trials were repeated. A successful trial was one in which the run appeared relaxed, the speed was within the required range, and there was no observed adjustment for plate contact. Running speed was monitored using timing gates. Five successful running trials were recorded per recruit, followed by a standing trial, in which recruits stood in a relaxed position with legs shoulder-width apart. This allowed for adjustment of dynamic joint angles relative to the neutral standing position of each recruit, providing anatomically relevant data. This entire process was completed both pre- and postactivity. Post-activity data collection took place as soon as possible after completion of the activity. Recruits finished the training activity at the location of data collection and immediately removed their additional load and changed into shorts and t-shirts. The time between recruits completing the activity and starting running trials was monitored and recorded (range: 10-84 min, mean (sd): 44 (21) min). Changes in plantar loading as a result of the training activity are likely to reduce with increased recovery time, such that all data were collected within 90 minutes of completion of the activity, based on existing findings [20], acknowledging that fatigue was induced in a 
different way in this study. Energy and water intake were not controlled upon completion of the activity, as these factors were not controlled during the activity.

Kinematic data were analysed using a three-dimensional model [21,22]. Ankle dorsiflexion was characterised by calculation of touchdown angle, peak angle, range of motion from touchdown to peak, time of peak dorsiflexion as a percentage of ground contact time and the angular velocity from touchdown to peak dorsiflexion. Ground contact time was determined from pressure data. The sagittal plane angle between the foot and the ground at touchdown was also calculated to provide an indicator of foot strike modality. Kinematic variables were analysed using customised MATLAB scripts (R2012a, The MathWorks Inc. Natick, MA, USA).

Pressure trials were exported from the Footscan software (RSscan International, Version 7). The software automatically divides the foot into zones (Figure 1), representing the hallux, lesser toes, five metatarsals, the midfoot, and the medial and lateral heel. Peak pressure, time of peak pressure, and impulse under each zone were evaluated. The foot was further divided into rearfoot (medial and lateral heel), midfoot and forefoot (hallux, lesser toes and metatarsals) regions. The relative contribution to total ground contact area (contact \%) under each of these regions, and their relative impulses (\%) were also examined. 


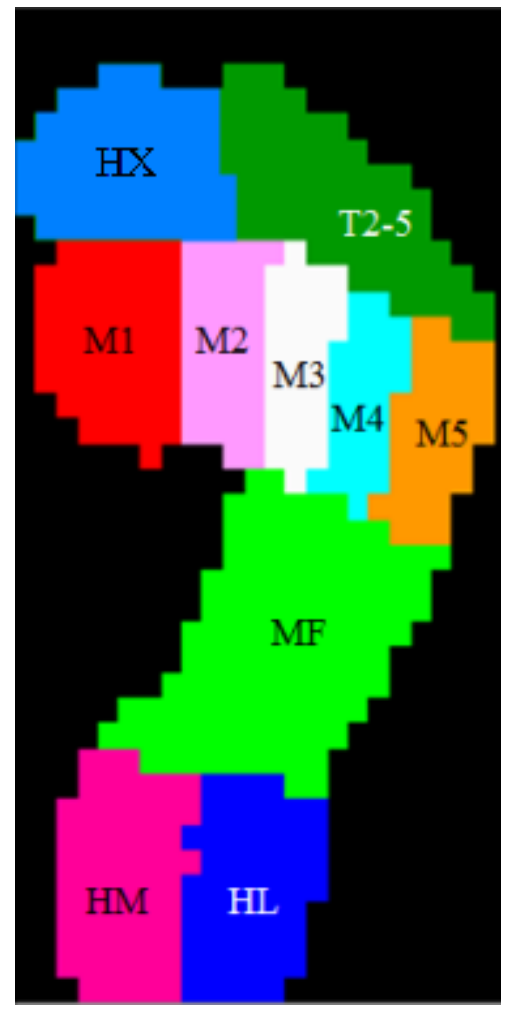

Figure 1: Plantar zone divisions

HX: Hallux; T2-5: lesser toes; M1-M5; first to fifth metatarsals; MF: midfoot; HM: medial heel; HL: lateral heel

All statistical analyses were undertaken using SPSS for Windows (Version 16.0, SPSS Inc., Chicago, IL, USA) with a statistical significance of $P<0.05$. To determine whether pressure variables should be normalised to body weight, bivariate correlation analyses between these variables were conducted. No variables were significantly associated with body weight either pre- or post-activity, thus pressure variables were not normalised to body weight. Normality was assessed for all variables by histogram observation and assessment of skewness and kurtosis values $(|z|<1.96$ indicated normality). Data from one randomly selected leg was assessed for each recruit. Means were compared between pre- and post-activity using paired t-tests (normally distributed variables) and Wilcoxon-signed rank tests (not normally distributed 
variables). Two-tailed values were considered for all variables. Effect sizes (d) [23] were reported for variables which differed between conditions $(P<0.05)$.

\section{Results}

Pressure data are presented in Table 1. Post-activity, there was a reduction in peak pressure and impulse under the hallux, lesser toes and first and second metatarsals. Peak pressure occurred earlier under the hallux and lesser toes, and later under the second-to-fifth metatarsals post-activity. There was no change in ground contact time. There was increased impulse and contact under the rearfoot, and a corresponding decrease under the forefoot post- compared with pre-activity. 
Table 1: Pressure variables pre- and post- 12-mile load carriage activity.

\begin{tabular}{|c|c|c|c|c|c|}
\hline & \multirow{2}{*}{\multicolumn{2}{|c|}{ Mean (sd) }} & \multirow{3}{*}{$\mathbf{P}$} & \multirow{3}{*}{ d } \\
\hline & & & & & \\
\hline & & Pre- & Post- & & \\
\hline & $\begin{array}{l}\text { Ground contact time } \\
(\mathrm{ms})\end{array}$ & $209(20)$ & $213(18)$ & 0.202 & \\
\hline \multirow{9}{*}{$\begin{array}{l}\text { Peak pressure } \\
\quad\left(\mathbf{N}^{\prime} \mathbf{c m}^{-2}\right)\end{array}$} & Hallux & $11.27(5.45)$ & $6.04(3.86)$ & $P<0.001$ & 1.08 \\
\hline & Lesser toes & $2.93(1.28)$ & $1.59(1.04)$ & $P<0.001$ & 1.14 \\
\hline & $1^{\text {st }}$ metatarsal & $16.95(6.17)$ & $10.42(5.22)$ & $P<0.001$ & 0.87 \\
\hline & $2^{\text {nd }}$ metatarsal & $21.98(5.70)$ & $17.75(4.35)$ & 0.002 & 0.64 \\
\hline & $3^{\text {rd }}$ metatarsal & $25.22(8.82)$ & $22.59(8.49)$ & 0.115 & \\
\hline & $4^{\text {th }}$ metatarsal & $25.31(12.23)$ & $23.15(9.58)$ & 0.314 & \\
\hline & $5^{\text {th }}$ metatarsal & $15.40(7.80)$ & $15.99(6.88)$ & 0.618 & \\
\hline & Medial heel & $15.48(6.63)$ & $15.98(6.92)$ & 0.728 & \\
\hline & Lateral heel & $15.53(8.15)$ & $15.34(9.37)$ & 0.773 & \\
\hline \multirow{9}{*}{ Impulse (N.s) } & Hallux & $11.09(8.04)$ & $4.71(3.84)$ & $P<0.001$ & 0.95 \\
\hline & Lesser toes & $2.61(2.30)$ & $1.27(1.43)$ & $P<0.001$ & 0.88 \\
\hline & $1^{\text {st }}$ metatarsal & $17.90(6.29)$ & $11.01(6.79)$ & $P<0.001$ & 0.97 \\
\hline & $2^{\text {nd }}$ metatarsal & $23.81(6.82)$ & $19.19(6.33)$ & 0.003 & 0.59 \\
\hline & $3^{\text {rd }}$ metatarsal & $24.04(8.03)$ & $21.76(8.17)$ & 0.197 & \\
\hline & $4^{\text {th }}$ metatarsal & $20.62(8.95)$ & $20.65(9.14)$ & 0.988 & \\
\hline & $5^{\text {th }}$ metatarsal & $12.60(6.93)$ & $13.00(6.60)$ & 0.649 & \\
\hline & Medial heel & $10.01(6.14)$ & $11.54(8.19)$ & 0.300 & \\
\hline & Lateral heel & $6.74(5.13)$ & $7.43(6.11)$ & 0.533 & \\
\hline \multirow{9}{*}{$\begin{array}{c}\text { Time of peak } \\
\text { pressure } \\
\text { (\% stance) }\end{array}$} & Hallux & $65.36(6.86)$ & $57.36(17.38)$ & 0.011 & 0.52 \\
\hline & Lesser toes & $59.60(15.80)$ & $42.53(21.62)$ & $P<0.001$ & 0.94 \\
\hline & $1^{\text {st }}$ metatarsal & $51.97(6.78)$ & $51.28(5.78)$ & 0.575 & \\
\hline & $2^{\text {nd }}$ metatarsal & $54.90(4.86)$ & $59.18(5.27)$ & $P<0.001$ & 0.86 \\
\hline & $3^{\text {rd }}$ metatarsal & $53.56(5.25)$ & $56.32(4.95)$ & 0.018 & 0.47 \\
\hline & $4^{\text {th }}$ metatarsal & $47.33(6.41)$ & $52.63(5.90)$ & $P<0.001$ & 0.74 \\
\hline & $5^{\text {th }}$ metatarsal & $42.04(10.30)$ & $48.37(9.24)$ & $P<0.001$ & 0.96 \\
\hline & Medial heel & $10.38(5.98)$ & $12.96(6.93)$ & 0.065 & \\
\hline & Lateral heel & $8.03(3.30)$ & $8.99(4.77)$ & 0.325 & \\
\hline \multirow{3}{*}{$\begin{array}{c}\text { Contact } \\
\text { percentage (\%) }\end{array}$} & Rearfoot & $20.81(2.60)$ & $22.44(2.42)$ & $P<0.001$ & 0.68 \\
\hline & Midfoot & $21.19(4.46)$ & $23.31(4.96)$ & 0.06 & \\
\hline & Forefoot & $58.54(5.52)$ & $55.11(5.22)$ & $P<0.001$ & 0.97 \\
\hline \multirow{3}{*}{$\begin{array}{c}\text { Relative } \\
\text { impulse (\%) }\end{array}$} & Rearfoot & $10.56(5.42)$ & $12.69(5.65)$ & 0.028 & 0.42 \\
\hline & Midfoot & $5.02(1.51)$ & $6.10(2.24)$ & $P<0.001$ & 0.74 \\
\hline & Forefoot & $84.23(5.53)$ & $81.21(6.44)$ & 0.001 & 0.50 \\
\hline
\end{tabular}

Note: rows highlighted in bold indicate variables which significantly differed post- compared with preactivity 
Kinematic data are presented in Table 2. There was no change in the angle between the foot and the ground, with the toes raised relative to the heel at touchdown in both conditions, indicative of a rearfoot strike. There was no difference in dorsiflexion angle at touchdown, or dorsiflexion velocity post-activity. There was an increase in peak dorsiflexion angle and dorsiflexion range of motion post- compared with pre-activity.

Table 2: Kinematic variables pre- and post- 12-mile load carriage activity.

\begin{tabular}{|c|c|c|c|c|c|}
\cline { 3 - 6 } \multicolumn{2}{c|}{} & \multicolumn{2}{c|}{ Mean (sd) } & & \\
\cline { 3 - 6 } \multicolumn{2}{c|}{} & Pre- & Post- & P & d \\
\hline Foot-ground angle & Foot Angle at TD $\left(^{\circ}\right)$ & $2.67(3.99)$ & $2.40(2.37)$ & 0.961 & \\
\hline \multirow{4}{*}{ Ankle dorsiflexion } & Touchdown ( $\left.{ }^{\circ}\right)$ & $2.49(4.66)$ & $0.69(6.14)$ & 0.343 & \\
\cline { 2 - 6 } & Peak ( ) & $\mathbf{- 1 0 . 4 8 ( 3 . 8 3 )}$ & $\mathbf{- 1 5 . 3 4 ( 5 . 1 6 )}$ & $\mathbf{0 . 0 0 1}$ & $\mathbf{0 . 9 5}$ \\
\cline { 2 - 6 } & Time of peak (\%) & $46.62(5.91)$ & $50.78(8.07)$ & 0.075 & \\
\cline { 2 - 6 } & Range of motion ( $\left.{ }^{\circ}\right)$ & $\mathbf{1 3 . 2 8 ( 3 . 1 2 )}$ & $\mathbf{1 6 . 1 5 ( 4 . 8 1 )}$ & $\mathbf{0 . 0 3 1}$ & $\mathbf{0 . 5 4}$ \\
\cline { 2 - 6 } & Velocity ( ${ }^{\circ}$. sec $\left.^{-1}\right)$ & $137.93(24.06)$ & $138.96(28.61)$ & 0.910 & \\
\hline
\end{tabular}

Note: $\mathrm{TD}=$ touchdown; rows highlighted in bold indicate variables which significantly differed postcompared with pre-activity. All angles are reported relative to the standing position, such that zero degrees of dorsiflexion would be reported in the standing trial. A negative dorsiflexion angle indicates a dorsiflexed ankle relative to standing. A positive foot angle at TD indicates that the toes are raised relative to the heel, typical of a heel strike running style.

\section{Discussion}

This study assessed the influence of a Royal Marines military training activity on barefoot running gait. As hypothesised, there was reduced loading under the lesser toes and hallux following the training activity, which is consistent with previous findings $[11-13,15,16]$. Nagel et al. [13] have suggested that a reduced contribution of the toes to push-off increases the loading of the metatarsals, thereby increasing risk of stress fracture. However, contrary to the hypothesis and to existing findings [11-15], peak pressure was reduced under the first and second metatarsals, and was unchanged under the third to fifth metatarsals post-activity. Reduced metatarsal loading was previously observed following prolonged walking [16]. The reduction in loading under 
the first and second metatarsals, as well as the reduced forefoot loading and increased rearfoot loading, may be a strategy to reduce forefoot discomfort during running. This could also explain why reduced metatarsal loading was previously observed following four days of walking [16]. This earlier study found that $88 \%$ of participants reported foot complaints after the activity, of which $22 \%$ related to the forefoot. This may have been the result of increased metatarsal loading at an earlier stage of the activity, as observed after shorter duration running activities $[11,12,14,15]$. This could have led to discomfort and an altered gait in order to avoid uncomfortable loading of these regions. Assessment of this using in-field, in-shoe devices would provide valuable insight.

Reduced peak pressure under the forefoot (including the hallux and lesser toes) coincided with increased rearfoot and midfoot loading. This indicates a reduced transfer of load from rearfoot to forefoot during stance post-activity. These changes may be influenced by plantar flexor muscle fatigue, of which there was evidence following the same military training activity, when assessed during walking [24]. The ankle plantar flexors provide eccentric dorsiflexion control during mid-stance, such that fatigue of these muscles may also explain the increased dorsiflexion observed postactivity. These findings suggest an increased reliance on forwards inertia in late stance. Reduced plantar flexor muscle function was previously suggested as an explanation for reduced loading under the toes and hallux [25], as was observed in the present study. This has implications for metatarsal injury risk, as activation of the plantar flexor muscles can reduce metatarsal bending moments [26], whereas reduced activity of these muscles results in increased metatarsal strain [9]. 
Earlier peak pressures under the hallux and lesser toes post-activity is evidence of a reduced active contribution to push-off. Peak toe pressure occurred at $60 \%$ of stance pre-activity, which coincides with the usual time of forefoot push-off [27], whereas peak toe pressure occurred at $43 \%$ of stance post-activity. Later peak pressures under the metatarsals were observed post-activity, in support of the hypothesis for this variable. A later peak second metatarsal pressure during barefoot running was the strongest predictor of third metatarsal stress fracture risk in Royal Marines recruits [17]. This previous study found that in recruits who sustained a third metatarsal stress fracture, peak pressure occurred under the second metatarsal at $60 \%$ of stance, compared with $57 \%$ of stance in recruits who remained injury-free. In the present study, peak pressure occurred under the second metatarsal at $59 \%$ of stance post-activity, compared with $55 \%$ of stance pre-activity. The later peak pressures occurred closer to the time of push-off following the training activity. There may have been an increased leverage from the metatarsals at push-off due to a reduced contribution from the toes. The contribution of the metatarsals to propulsion during running stance may therefore be an important mechanism in the development of metatarsal stress fractures. Interventions to minimise the plantar loading changes observed following a military training activity could help to reduce the risk of metatarsal stress fracture. Footwear designed to facilitate the transfer of loading from rearfoot to forefoot, or strength training interventions of both the foot and ankle plantar flexor muscles warrant investigation.

Greater peak pressure under the heel was identified as a risk factor for tibial stress fractures in Royal Marines recruits [18]. This variable did not change post-activity, in contrast with the hypothesis. There was greater overall loading (rearfoot impulse and 
contact percentage) under the rearfoot following the activity, but this is not known to be a risk factor for tibial stress fracture. Further exploration of the mechanisms by which tibial stress fractures develop, and identification of training activities which increase this variable, is warranted.

The decision to use a barefoot running protocol in this study was based upon the recent identification of plantar loading variables as risk factors for stress fractures in this population, when using the same protocol $[17,18]$. Future investigation of changes in plantar loading during shod running and during load carriage whilst wearing military boots, would provide further insight into mechanisms for injury. It is important to note that although it was suggested that the plantar flexor muscles had reduced function post-activity in the present study, no direct measurements of muscular activity were obtained in the present study. This suggestion was based on findings following the same training activity but during walking [24]. Assessment of muscular activity during running following this training activity would strengthen these findings. Additionally, only male recruits were included in this study and the findings may not be generalisable to female military recruits. Similarly, foot type was not recorded in this study and it should be acknowledged that populations with a specific foot type may respond differently to completing a prolonged training activity.

\section{Conclusion}

A prolonged ( $150 \mathrm{~min}$ ) military training activity, with $35.5 \mathrm{~kg}$ of additional load, resulted in increased rearfoot loading, reduced forefoot loading and increased dorsiflexion during barefoot running. This is indicative of a reduced transfer of loading from rearfoot to forefoot during stance. Peak pressure under the second metatarsal 
occurred later following the training activity, a characteristic that has been identified as the strongest predictor of third metatarsal stress fracture risk in Royal Marines recruits when using the same barefoot running protocol. The reduced transfer of loading from rearfoot to forefoot following a military training activity may therefore contribute to the high incidence of metatarsal stress fractures in this population. Interventions intended to minimise these changes should be investigated. 


\section{Acknowledgements:}

The authors would like to thank recruits from the Commando Training Centre Royal Marines (CTCRM) who participated in the study. The authors would also like to acknowledge the assistance of CTCRM staff, in particular Richard Hales, Senior Physiotherapist at CTCRM. Finally, the authors would like to thank Dr Trish Davey and Sophie Britland from the Institute of Naval Medicine for their help in data collection. This work was part-funded by the Institute of Naval Medicine, Ministry of Defence.

\section{Conflict of Interest Statement}

We are not aware of any conflict of interest related to the manuscript and its publication.

\section{References}

[1] K.R. Kaufman, S. Brodine, R. Shaffer, Military training-related injuries: surveillance, research, and prevention, Am. J. Prev. Med. 18 (2000) 54-63.

[2] K. Munnoch, R.S. Bridger, Smoking and injury in Royal Marines' training, Occup. Med. 57 (2007) 214-216. doi:10.1093/occmed/kql170.

[3] K. Munnoch, The Psychological Impact of Physical Injury on Recovery in Royal Marines' Recruit Training., University of Southampton, 2008.

[4] S.A. Birrell, R.H. Hooper, R.A. Haslam, The effect of military load carriage on ground reaction forces, Gait Posture. 26 (2007) 611-614. doi:10.1016/j.gaitpost.2006.12.008.

[5] J. Knapik, K. Reynolds, J. Staab, J.A. Vogel, B. Jones, Injuries associated with strenuous road marching, Mil. Med. 157 (1992) 64-67.

[6] R.M. Orr, R. Pope, V. Johnston, J. Coyle, Soldier occupational load carriage: a narrative review of associated injuries, Int. J. Inj. Contr. Saf. Promot. 21 (2014) 388-396. doi:10.1080/17457300.2013.833944.

[7] R.A. Ross, A. Allsopp, Stress fractures in Royal Marines recruits, Mil. Med. 167 (2002) 560-565. 
[8] E.A. Arendt, Stress fractures and the female athlete, Clin. Orthop. (2000) 131138.

[9] A. Arndt, I. Ekenman, P. Westblad, A. Lundberg, Effects of fatigue and load variation on metatarsal deformation measured in vivo during barefoot walking, $\mathrm{J}$. Biomech. 35 (2002) 621-628.

[10] D.R. Carter, W.E. Caler, D.M. Spengler, V.H. Frankel, Fatigue Behavior of Adult Cortical Bone: The Influence of Mean Strain and Strain Range, Acta Orthop. 52 (1981) 481-490. doi:10.3109/17453678108992136.

[11] M. Bisiaux, P. Moretto, The effects of fatigue on plantar pressure distribution in walking, Gait Posture. 28 (2008) 693-698. doi:10.1016/j.gaitpost.2008.05.009.

[12] E. Escamilla-Martínez, A. Martínez-Nova, B. Gómez-Martín, R. SánchezRodríguez, L.M. Fernández-Seguín, The effect of moderate running on foot posture index and plantar pressure distribution in male recreational runners, J. Am. Podiatr. Med. Assoc. 103 (2013) 121-125.

[13] A. Nagel, F. Fernholz, C. Kibele, D. Rosenbaum, Long distance running increases plantar pressures beneath the metatarsal heads: a barefoot walking investigation of 200 marathon runners, Gait Posture. 27 (2008) 152-155. doi:10.1016/j.gaitpost.2006.12.012.

[14] R. Weist, E. Eils, D. Rosenbaum, The influence of muscle fatigue on electromyogram and plantar pressure patterns as an explanation for the incidence of metatarsal stress fractures, Am. J. Sports Med. 32 (2004) 18931898.

[15] T. Willems, R. De Ridder, P. Roosen, The effect of a long-distance run on plantar pressure distribution during running, Gait Posture. 35 (2012) 405-409. doi:10.1016/j.gaitpost.2011.10.362.

[16] N.M. Stolwijk, J. Duysens, J.W.K. Louwerens, N.L.W. Keijsers, Plantar pressure changes after long-distance walking, Med. Sci. Sports Exerc. 42 (2010) 22642272. doi:10.1249/MSS.0b013e3181e305f4.

[17] S. Dixon, M. Nunns, C. House, H. Rice, M. Mostazir, V. Stiles, J. Fallowfield, A. Allsopp, Prospective study of biomechanical risk factors for second and third metatarsal stress fractures in military recruits, J. Sci. Med. Sport. 0 (2018). doi:10.1016/j.jsams.2018.06.015.

[18] M. Nunns, C. House, H. Rice, M. Mostazir, T. Davey, V. Stiles, J. Fallowfield, A. Allsopp, S. Dixon, Four biomechanical and anthropometric measures predict tibial stress fracture: a prospective study of 1065 Royal Marines, Br. J. Sports Med. (2016) bjsports-2015-095394. doi:10.1136/bjsports-2015-095394.

[19] T. Willems, E. Witvrouw, A. De Cock, D. De Clercq, Gait-related risk factors for exercise-related lower-leg pain during shod running, Med. Sci. Sports Exerc. 39 (2007) 330-339. doi:10.1249/01.mss.0000247001.94470.21.

[20] T. Horita, P.V. Komi, C. Nicol, H. Kyröläinen, Effect of exhausting stretchshortening cycle exercise on the time course of mechanical behaviour in the drop jump: possible role of muscle damage, Eur. J. Appl. Physiol. 79 (1999) 160-167. doi:10.1007/s004210050490.

[21] R.W. Soutas-Little, G.C. Beavis, M.C. Verstraete, T.L. Markus, Analysis of foot motion during running using a joint co-ordinate system, Med. Sci. Sports Exerc. 19 (1987) 285-293.

[22] H. Rice, Potential Mechanisms for the Occurrence of Tibial Stress Fractures, Metatarsal Stress Fractures and Ankle Inversion Injuries in Royal Marine Recruits, Doctoral Thesis, University of Exeter, 2015. 
[23] J. Cohen, The Analysis of Variance, in: Stat. Power Anal. Behav. Sci., Second Edition, Lawrence Erlbaum Associates, 1988: pp. 148-150.

[24] H. Rice, J. Fallowfield, A. Allsopp, S. Dixon, Influence of a 12.8-km military load carriage activity on lower limb gait mechanics and muscle activity, Ergonomics. 60 (2017) 649-656. doi:10.1080/00140139.2016.1206624.

[25] L. Ferris, N.A. Sharkey, T.S. Smith, D.K. Matthews, Influence of extrinsic plantar flexors on forefoot loading during heel rise, Foot Ankle Int. 16 (1995) 464-473.

[26] N.A. Sharkey, L. Ferris, T.S. Smith, D.K. Matthews, Strain and loading of the second metatarsal during heel-lift, J. Bone Joint Surg. Am. 77 (1995) 10501057.

[27] A. De Cock, D. De Clercq, T. Willems, E. Witvrouw, Temporal characteristics of foot roll-over during barefoot jogging: reference data for young adults, Gait Posture. 21 (2005) 432-439. doi:10.1016/j.gaitpost.2004.05.004. 